Sara Gori

Nicola Morelli

Michelangelo Maestri

Monica Fabbrini

Erica Bonanni

Luigi Murri

\section{Sleep quality, chronotypes and preferential timing of attacks in migraine without aura}

Published online: 20 July 2005

S. Gori $(\varangle) \cdot$ N. Morelli • M. Maestri

M. Fabbrini • E. Bonanni • L. Murri

Institute of Neurology,

Department of Neurosciences,

University of Pisa,

Via Roma 67, I-56126 Pisa, Italy

e-mail: s.gori@med.unipi.it

Tel.: +39-050-993100/992443

Fax: +39-050-554808

\begin{abstract}
Clinical observations
show that migraine attacks have a seasonal, menstrual and circadian timing, suggesting a role of chronobiological mechanisms and their alterations in the disease, but little experimental data exists about this issue. The aim of this study was to estimate sleep quality chronotypes, and the possible circadian timing of attacks in migraneurs. One hundred patients suffering from migraine without aura according to the IHS criteria (2004), and 30 controls were enrolled. Morning and evening type subjects were more represented in migraine patients than in controls
\end{abstract}

and showed a tendency towards worse sleep quality and higher disability. Forty-two percent of migraineurs presented more than $75 \%$ of their attacks at night. Morning and evening types rather than intermediate and differences between real and preferred times may represent stressors that can worsen the disease. A preferential timing for occurrence of migraine attacks during the night and early morning hours was documented.

Key words Migraine - Chronotype • Chronobiological rhythms - Sleep • Wake

\section{Introduction}

The relationship between sleep and headache has been known for over a century. Headache and sleeping problems are both some of the most commonly reported problems in clinical practice, and cause considerable social and family problems, as well as socio-economic impact and costs. Much data suggests an association between headache, especially migraine, on one side and sleep and its disorders on the other, but the cause and effect of this relationship is not clear. With regard to migraine, rest and sleep usually bring relief and have therefore been attempted in the treatment strategy; on the other hand however, migraine episodes are frequently triggered by several fac- tors including sleep pattern changes; emotional stress, hypoglycaemia, sensorial stimulation (loud noise, bright light, heat or cold) and also lack of sleep or excess (weekend migraine) may, indeed, represent migraine triggers. Premonitory symptoms like mood states such as alert, tense, depressed or tired and changes in sleep quality have been described to occur up to 2 days before a migraine attack and were hypothesised to be related to a hypothalamic involvement in the prodromic phase of migraine [1]. Recently it has also been documented that overuse of migraine symptomatic drugs may worse sleep pattern in migraineurs and the withdrawal of the misused medication can alleviate the associated sleep disturbance [2].

Several findings also suggest a role of chronobiological factors in migraine, probably related - as previously 
reported - to a hypothalamic involvement [3]. Clinical observations show, indeed, that migraine attacks have a seasonal, menstrual and circadian timing, suggesting a role of chronobiological mechanisms and their alterations in the disease, but little experimental data exists on this issue. In particular, a single study carried out on 1698 migraine patients including 3582 attacks documented that about $48 \%$ of the attacks happened between 4 and 9 a.m. [4], and a recent review [5] underlines as very sparse and insufficient information regard early morning headache and sleep.

The aim of this study was to estimate migraineurs' sleep quality, chronotypes and the possible circadian timing of attacks, and to evaluate their possible relationship with psychiatric comorbidity and clinical parameters.

\section{Materials and methods}

One hundred patients (mean age \pm S.D. $38.6 \pm 10.4$ years; range 23-50 years) suffering from migraine without aura according to the IHS criteria [6] were enrolled in the study. Thirty control subjects (mean age \pm S.D. $37.1 \pm 13$ years; range $23-60$ years) represented the control group. Chronotypes were evaluated using the Italian version of the reduced Morningness-Eveningness Questionnaire (rMEQ) [7]; it includes five questions exploring subjective preference for the time to fall asleep, awakening and period of the day with highest satisfaction for wellbeing and allows classification of patients into morning, evening and intermediate type subjects according to different scores. rMEQ score values ranging from 18 to 25 identify morningness, scores ranging between 12 and 17 identify intermediate type, and finally lower values on rMEQ score (4-11) indicate eveningness. Sleep quality (by means of the Pittsburgh Sleep Quality Index (PSQI)) and symptoms of depression or anxiety (using the Beck Depression Index (BDI) and State Trait Anxiety Index (STAI)) were also evaluated. The actual time of falling asleep and awakening were obtained from subjective evaluation included in the PSQI.

In the migraine group, length of the disease, migraine attack frequency and disability using the Migraine Disability Assessment Scale (MIDAS) were considered.

\section{Results}

Poor subjective sleep quality, evaluated by means of the PSQI (i.e., PSQI score >5) was documented in $64 \%$ of patients with migraine and in $33 \%$ of the control group. Mean migraine group PSQI score value significantly differed from controls (patients vs. controls $7 \pm 4.2 v s$. $3.75 \pm 1.3 ; p<0.01$ ); concerning the different items included in the PSQI, the greatest difference regards sleep latency (patients vs. controls $25.1 \pm 18.9 \mathrm{~min}$ vs. 10 \pm 5.2 ; $p<0.01)$. PSQI scores correlated with both BDI values $(p<0.01)$ and STAI values $(p<0.05)$, whereas they failed to correlate with clinical parameters such as duration of disease, mean monthly frequency of migraine attacks and disability (i.e., MIDAS score).

According to the BDI scores, patients were divided into two groups: the first with BDI value $>10$ (the so-called depressed group, DG) and the second with a value $\leq 10$ (non-depressed group, NDG); poor sleep quality with percentages greater than in controls was documented in both groups with a percentage of $85 \%$ in DG and $50 \%$ in NDG.

With regard to chronotype distributions, the intermediate type was less represented in the migraine group than in controls (44\% of patients and $73 \%$ of controls), whereas morning and evening type subjects were more represented in the migraine patients than in controls. Evening types accounted for $16 \%$ of migraineurs and $13 \%$ of control subjects, while morning type represented $28 \%$ of migraine patients and $14 \%$ of controls. In both DG and NDG the intermediate type percentage was smaller than in the control group, either evening and morning types being more represented.

Among patients, morning and evening types, compared to intermediate types, showed a tendency towards a worse sleep quality, and a higher MIDAS score $(p<0.05)$.

Moreover, according to clinical diaries referring to the last 3 months, $42 \%$ of migraineurs presented more than $75 \%$ of their attacks at night and in the early morning hours, especially from 3 to 7 a.m.

\section{Discussion}

Morning and evening types rather than intermediate were better represented among migraineurs in comparison with control group subjects and differences between real and preferred times to fall asleep and to awake were longer than in controls.

As morning and evening types showed poorer sleep quality and higher disability, at least as assessed by means of MIDAS, it might be hypothesised that extreme chronotypes and the differences between real and preferred time to fall asleep and to awake, they experimented because of social rhythms, may represent stressors that worsen clinical presentation of the disease; in other words, chronobiological aspects might interfere with clinical phenotype in migraine. Desynchronisation between biological endogenous clock and lifestyle may promote stress and account for different levels of severity in migraine patients.

It might be underlined that data regarding chronotype in this study were obtained only with a validated questionnaire, so they need to be replicated and confirmed; how- 
ever, from a speculative point of view, they might support the hypothesis of an impairment of chronobiological rhythms occurring in migraine; an alteration in melatonin secretion, which plays a main role in circadian rhythm organisation, has been, indeed, observed in migraine concerning both quantitative aspects and phase [8,9].

Our findings also confirm previous data from Fox and Davis [4] concerning a preferential timing for occurrence of migraine attacks during the night and early morning hours in a relevant percentage of patients. The data in the literature up to now have not allowed an explanation of the preferential occurrence of migraine without aura in the second part of the night and in the early morning, because it may depend on a non better defined impairment of chronobiological mechanisms or be related to a temporal relationship with sleep stages; an emergence of migraine attacks in REM sleep has, indeed, been polygraphically documented in the past few years [10]. Further studies should focus on night-time and early morning migraine headache to evaluate its physiopathological aspects and also to verify if the preferential time of occurrence may affect response to migraine therapy.

\section{References}

1. Spierings EL, Sorbi M, Haimowitz BR, Tellegen B (1996) Changes in daily hassles, mood, and sleep in the 2 days before a migraine headache. Clin J Pain 12(1):38-42

2. Hering-Hanit R, Yavetz A, Dagan Y (2000) Effect of withdrawal of misused medication on sleep disturbances in migraine sufferers with chronic daily headache. Headache 40(10):809-812

3. Dodick DW, Eross EJ, Parish JM (2003) Clinical, anatomical, and physiologic relationship between sleep and headache. Headache 43(3):282-292
4. Fox AW, Davis RL (1998) Migraine chronobiology. Headache 38(6):436-441

5. Jennum P, Jensen R (2002) Sleep and headache. Sleep Med Rev 6:471-479

6. (2004) The International Classification of Headache Disorders. Headache Classification Committee of the International Headache Society. Cephalalgia 24:1-160

7. Natale V (1999) Validazione di una scala ridotta di Mattutinità (rMEQ). Boll Psicol Appl 229:19-26
8. Gagnier J (2001) The therapeutic potential of melatonin in migraines and other headache types. Altern Med Rev 6(4):383-389

9. Peres MF, Sanchez del Rio M, Seabra ML, Tufik S, Abucham J, Cipolla-Neto J, Silberstein SD, Zukerman E (2001) Hypothalamic involvement in chronic migraine. J Neurol Neurosurg Psychiatry 71(6):747-751

10. Drake ME Jr, Pakalnis A, Andrews JM, Bogner JE (1990) Nocturnal sleep recording with cassette EEG in chronic headaches. Headache 30(9):600-603 